\title{
Optimal design of electromagnetic cloaks with multiple dielectric materials by topology optimization
}

\section{$\operatorname{AUTHOR}(\mathrm{S})$ :}

Kishimoto, Naoki; Izui, Kazuhiro; Nishiwaki, Shinji; Yamada, Takayuki

\section{CITATION:}

Kishimoto, Naoki ... [et al]. Optimal design of electromagnetic cloaks with multiple dielectric materials by topology optimization. Applied Physics Letters 2017, 110(20): 201104.

ISSUE DATE:

2017-05-15

URL:

http://hdl.handle.net/2433/224941

\section{RIGHT:}

(c) 2017 AIP Publishing. This article may be downloaded for personal use only. Any other use requires prior permission of the author and AIP Publishing. The following article appeared in 'Appl. Phys. Lett. 110, 201104 (2017)' and may be found at http://aip.scitation.org/doi/10.1063/1.4983715/ The full-text file will be made open to the public on 15 May 2018 in accordance with publisher's 'Terms and Conditions for Self-Archiving'. 
Optimal design of electromagnetic cloaks with multiple dielectric materials by topology optimization

Naoki Kishimoto, Kazuhiro Izui, Shinji Nishiwaki, and Takayuki Yamada

Citation: Appl. Phys. Lett. 110, 201104 (2017); doi: 10.1063/1.4983715

View online: http://dx.doi.org/10.1063/1.4983715

View Table of Contents: http://aip.scitation.org/toc/apl/110/20

Published by the American Institute of Physics

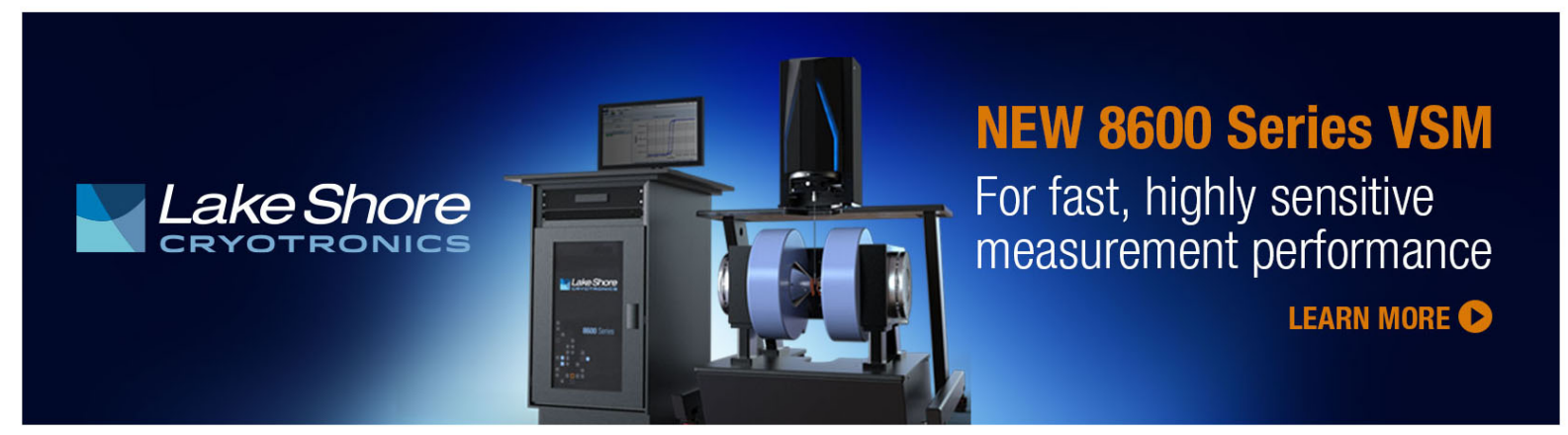




\title{
Optimal design of electromagnetic cloaks with multiple dielectric materials by topology optimization
}

\author{
Naoki Kishimoto, Kazuhiro Izui, Shinji Nishiwaki, and Takayuki Yamada ${ }^{a}$ \\ Department of Mechanical Engineering and Science, Kyoto University, 615-8504 Kyoto, Japan
}

(Received 10 April 2017; accepted 1 May 2017; published online 16 May 2017)

\begin{abstract}
This paper presents an optimal design method for electromagnetic cloaks composed of multiple dielectric materials based on a topology optimization. To obtain optimal configurations that are perfectly free from intermediate materials, so-called grayscales, we apply a Multi-Material Level Set topology description model. The level set functions that are design variables are updated using topological derivatives that also function as design sensitivities, and we derive the topological derivatives for multiple materials. Three numerical examples demonstrate the validity of the proposed method and the advantage of using multiple materials. Published by AIP Publishing.
\end{abstract}

[http://dx.doi.org/10.1063/1.4983715]

In recent years, developments in the field of material chemistry have spurred investigations of various electromagnetic metamaterials. ${ }^{1-3}$ As applied metamaterial devices, electromagnetic cloaks that can make an object unobservable by wrapping it have attracted the attention of many researchers. The theory of electromagnetic cloaks was first proposed by Pendry and Leonhardt independently. ${ }^{4,5}$ Motivated by the pioneering works, there have been further investigations of electromagnetic cloaking devices. ${ }^{6-11}$ The theory used in these studies is based on the concept of bending a path through which electromagnetic waves pass, using coordinate transformation, but it requires materials with spatially varying and infinite permittivity and permeability. Such extremely complex material parameters do not exist in nature and are very challenging to realize. Experiments using metamaterials that have periodic structures have been carried out based on this theory, ${ }^{12-14}$ but performances were far from ideal.

On the other hand, a topology optimization method that realizes cloaking by placing a simple isotropic material around the object was reported. ${ }^{15-20}$ Topology optimization methods, ${ }^{21}$ the most flexible type of structural optimization method, have been applied in many design problems for electromagnetic devices. Andkjær and Sigmund applied a density-based topology optimization for the design of an electromagnetic cloak made of a single-property dielectric material. ${ }^{15}$ However, the optimized configuration using this method included grayscales, areas of intermediate density between that of air and the dielectric material. The presence of grayscales makes practical fabrication problematic since a physical interpretation of such areas is elusive. Andkjær et al. also applied a density-based topology optimization with a filtering technique to obtain a graded optimal structure. ${ }^{19}$ Heo and Yoo proposed a design method for microwave cloaking considering multiple directions using a density-based phase filed method for the topology optimization. ${ }^{20}$ Although grayscales are restricted near the interfaces in these density-based approaches, perfectly clear boundaries are not obtained. That is, the optimal configurations are obtained based on the assumption that intermediate materials exist.
To overcome the problem of grayscales, level set basedtopology optimization methods have been proposed ${ }^{22}$ and applied for the design of cloaks. ${ }^{16-18}$ An experimental demonstration of the obtained optimal configuration was also presented. ${ }^{23}$ These examples have been shown to have ideal cloaking performance under limited conditions, but previous studies on the design of electromagnetic cloaking devices have been based on methods applied to a single material. Despite the fact that single-material designs have limits beyond which performance cannot be improved, a design method for multiple materials has not yet been proposed. Consequently, sufficient optical cloaking under demanding conditions, for example, for multiple wavelengths and multiple incident angles, is seldom obtained. However, by using multiple materials, design flexibility beyond that of conventional design methods can be achieved, leading to further improvements in cloaking performance.

Therefore, we present the optimal design of optical cloaks with multiple dielectric materials using the level setbased topology optimization method. First, we construct a design method for dealing with multiple dielectric materials and formulate the optimal design problem. Next, we show optimized designs of optical cloaks to confirm the validity of the proposed method and demonstrate the advantage of using multiple materials.

We adopt the MM-LS (Multi-Material Level Set) model for representing the distributions of multiple materials. ${ }^{24}$ The MM-LS model uses a total of $n$ level set functions for $n$ materials, plus the void phase. The advantages of the MMLS model are that clear boundaries between materials are obtained and the number of materials to be used can be increased systematically, as follows:

$$
\begin{cases}\text { Air } & : \psi_{n}^{0}=\left\{1-\chi\left(\phi^{1}\right)\right\}, \\ \quad \vdots & \\ \text { Material } k & : \psi_{n}^{k}=\left\{1-\chi\left(\phi^{k+1}\right)\right\} \prod_{i=1}^{k} \chi\left(\phi^{i}\right), \\ (1 \leq k \leq n-1) & \\ \text { Material } n & : \psi_{n}^{n}=\prod_{i=1}^{n} \chi\left(\phi^{i}\right) .\end{cases}
$$

a)takayuki@me.kyoto-u.ac.jp 
Here, the position $\boldsymbol{x}$ where the function $\psi_{n}^{k}(\boldsymbol{x})$ has a value of 1 is occupied by the $k$-th material and $\chi\left(\phi^{i}(\boldsymbol{x})\right)$ is a characteristic function defined as

$$
\chi\left(\phi^{i}(\boldsymbol{x})\right)= \begin{cases}1 & \text { if } \phi^{i}(\boldsymbol{x}) \geq 0 \\ 0 & \text { if } \phi^{i}(\boldsymbol{x})<0\end{cases}
$$

Because topology optimization problems are ill-posed, regularization or relaxation is required. Based on the formulation of the level set-based topology optimization method, ${ }^{22}$ the objective functional $F$ is regularized here by adding a fictitious interface energy term, as follows:

$$
F_{\text {regularized }}\left(\psi_{n}^{k}\right)=F\left(\psi_{n}^{k}\right)+\sum_{k=1}^{n} \int_{\Omega_{\text {design }}} \frac{1}{2} \tau^{k}\left|\nabla \phi^{k}\right|^{2} .
$$

$\Omega_{\text {design }}$ is the object domain in which the object to be designed resides. The second term on the right side of Eq. (3) is a regularization term for the multiple material problem.

Directly finding level set functions that minimize $F_{\text {regularized }}$ is problematic, and so, we introduce a fictitious time $t$ and assume that the variation in the level set functions with respect to time $t$ is proportional to the design sensitivity $F_{\text {regularized }}^{(k)}$ for the $k$-th level set function $\phi^{k}$, as follows:

$$
\begin{aligned}
\frac{\partial \phi^{k}}{\partial t} & =-F_{\text {regularized }}^{(k)} \\
& =-\mathscr{D} \mathscr{F}^{(k)}+\tau^{k} \nabla^{2} \phi^{k} .
\end{aligned}
$$

We note that the above equation is a type of reactiondiffusion equation, and the derivative of the objective functional $\mathscr{D} \mathscr{F}^{(k)}$ is defined as the "topological derivative" 25 for the $k$-th level set function. In the MM-LS model, this derivative is given by

$$
\mathscr{D}^{(k)}=\sum_{i=k}^{n} \psi_{n}^{i} \mathscr{D}^{i \rightarrow(k-1)}+\psi_{n}^{(k-1)} \sum_{i=k}^{n} \frac{\partial \psi_{n}^{i}}{\partial \psi_{k}^{k}} \mathscr{D F}^{(k-1) \rightarrow i},
$$

where $\mathscr{D} \mathscr{F}^{i \rightarrow j}$ is the topological derivative when material $i$ changes to material $j$, as illustrated in Fig. 1. In this paper, $\mathscr{D}_{\mathscr{F}}^{i \rightarrow j}$ is defined as follows:

$$
\mathscr{D} \mathscr{F}^{i \rightarrow j}:=\lim _{\varepsilon \rightarrow 0} \frac{\left\{\left(F+\delta F^{i \rightarrow j}\right)-F\right\} \operatorname{sign}\left(\delta \phi^{k}\right)}{\left|\operatorname{meas}\left(\Omega \backslash \Omega_{\varepsilon}\right)-\operatorname{meas}(\Omega)\right|} .
$$

$\Omega$ is an object domain, and $\Omega_{\varepsilon}$ is a circle of radius $\varepsilon . \delta \phi^{k}$ is the amount of variation in the $k$-th level set function when material $i$ occupying $\Omega_{\varepsilon}$ changes to material $j . \delta F^{i \rightarrow j}$ is the amount of variation in the objective functional.

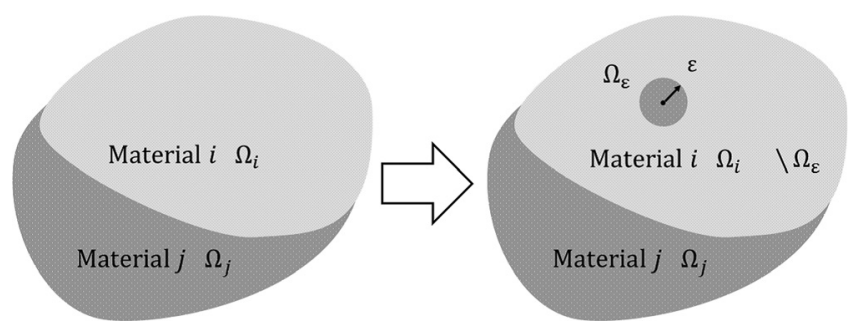

FIG. 1. Concept of topological derivative $\mathscr{D} \mathscr{F}^{i \rightarrow j}$.
We now consider the design of a two-dimensional electromagnetic cloak device for TE (Transverse Electric) waves based on the model shown in Fig. 2. The governing equation follows the Helmholtz equation for a polarized electric field $E_{z}$.

$$
\nabla \cdot\left(\mu_{r}^{-1} \nabla E_{z}\right)+k_{0}^{2} \varepsilon_{r} E_{z}=0,
$$

where $\varepsilon_{r}, \mu_{r}$, and $k_{0}$ are the relative permittivity, relative permeability, and wave number in a vacuum, which is expressed as $k_{0}=\omega \sqrt{\varepsilon_{0} \mu_{0}}$, respectively. $\varepsilon_{0}$ and $\mu_{0}$ are the electric permittivity and magnetic permeability in a vacuum, respectively. The radii of the inner, middle, and outer domains are set to $0.1,0.4$, and $1.0 \mathrm{~m}$, respectively. The circular disc at the center of domain $\Gamma_{\mathrm{PEC}}$ is set as a perfect electric conductor (PEC) to represent the scattering object that is to be hidden by the cloak. Here, incident plane waves $E_{z}^{\text {inc }}$ enter the scattering domain from the left side

$$
E_{z}^{\mathrm{inc}}=E_{0} e^{-j k_{0} k \cdot r},
$$

where $E_{0}$ is the amplitude of the incident waves, $j=\sqrt{-1}$, $\boldsymbol{k}=\left(k_{x}, k_{y}\right)^{T}$ is the normalized directional wave vector, and $\boldsymbol{r}=(x, y)^{T}$ is the position vector. The gray domain, $\Omega_{\text {design }}$, is the fixed design domain in which a material configuration consisting of dielectric materials and air is distributed. The domain exterior to the fixed design domain, $\Omega_{\text {out }}$, is the scattering domain, and a first order absorbing boundary condition is imposed on the outer boundary, $\Gamma_{\mathrm{ABC}}$.

In this paper, the purpose of the optimization problem is to find the configuration of dielectric materials in the cloak domain which makes the electromagnetic waves propagate as if the scattering object does not exist. Therefore, the objective function is set to minimize the norm of the difference between the electric field and a reference field, namely the electric field when no scattering object is present, as follows:

$$
\inf _{\phi^{k}} F\left(\psi_{n}^{k}\right)=\int_{\Omega_{\text {out }}}\left(E_{z}-E_{z}^{\mathrm{ref}}\right)^{*}\left(E_{z}-E_{z}^{\mathrm{ref}}\right) \mathrm{d} \Omega,
$$

subject to Governing equation,

Boundary conditions,

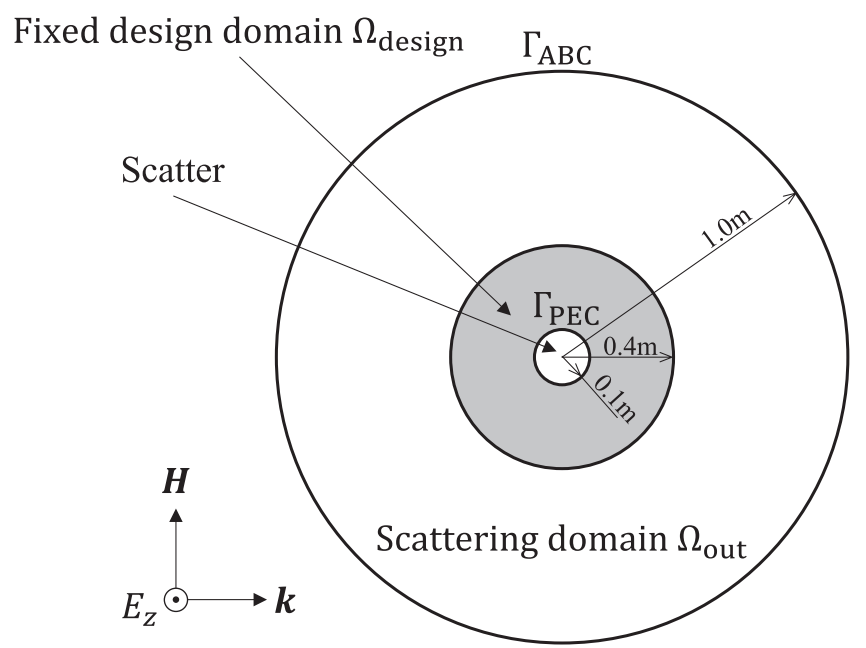

FIG. 2. Analysis domain and boundary conditions for the cylindrical cloak design problem. 
where $E_{z}^{\text {ref }}$ is the reference field and * denotes a complex conjugate. We note that the objective function is equivalent to minimizing the scattered field in the scattering domain. ${ }^{15}$ $\varepsilon_{r}$ is an extended relative permittivity defined using the characteristic function $\psi_{n}^{k}$ as follows:

$$
\varepsilon_{r}\left(\psi_{n}^{k}\right)=\sum_{k=0}^{n} \varepsilon_{r}^{k} \psi_{n}^{k},
$$

where $\varepsilon_{r}^{k}$ and $\varepsilon_{r}^{0}$ are the relative permittivity of the $k$-th dielectric material and air, respectively. We restrict our investigations to nonmagnetic materials, and so, relative permeability $\mu_{r}$ is set to 1.0 .

Next, we explain the topological derivative required for the optimization process. We derive the topological derivative by using the adjoint variable method. The topological derivative in the TE mode is as follows: ${ }^{26}$

$$
\mathscr{D} \mathscr{F}^{i \rightarrow j}=2 \Re\left[k_{0}^{2}\left(\varepsilon_{r}^{i}-\varepsilon_{r}^{j}\right) E_{z} \tilde{E}_{z}\right] .
$$

$\tilde{E}_{z}$ is a solution to the adjoint problem defined below:

$$
\begin{gathered}
\nabla^{2} \tilde{E}_{z}+k_{0}^{2} \varepsilon_{r}\left(\psi_{n}^{k}\right) \tilde{E}_{z}=0 \quad \text { in } \Omega_{\text {design }}, \\
\nabla^{2} \tilde{E}_{z}+k_{0}^{2} \varepsilon_{r}^{\text {air }} \tilde{E}_{z}=\left(E_{z}-E_{z}^{\text {ref }}\right)^{*} \quad \text { in } \Omega_{\text {out }}, \\
\boldsymbol{n} \cdot \nabla \tilde{E}_{z}=0 \text { on } \Gamma_{\mathrm{PEC}} \\
\tilde{E}_{z}{ }^{i}=\tilde{E}_{z}{ }^{j} \text { on } \Gamma_{i j}, \\
\nabla \cdot \tilde{E}_{z}{ }^{i}=\nabla \cdot \tilde{E}_{z}{ }^{j} \text { on } \Gamma_{i j} .
\end{gathered}
$$

where $\boldsymbol{n}$ is an outward-pointing normal vector. We note that $\Gamma_{i j}$ is the boundary between material $i$ and material $j$.

Here, we apply our method to the cylindrical cloak design problem shown in Fig. 2. First, we confirm the validity of our proposed method through Example 1, which is the most fundamental problem setting. Next, we show the advantage of using multiple materials through Examples 2 and 3 .

The relative permittivity of the dielectric materials and air is set as 2.0, 1.5, and 1.0. The magnitude of the regularization parameters, $\tau^{1}$ and $\tau^{2}$, is set to $1.0 \times 10^{-4} . E_{0}$ is set as $1000 \mathrm{~V} / \mathrm{m}$.

First, the results for Example 1 are shown. The operating frequency is set as $2.5 \mathrm{GHz}$. Figure 3(a) shows the optimized configuration of the dielectric materials. Red and green domains indicate the materials with relative permittivities of 2.0 and 1.5 , respectively, and blue indicates air. We confirm

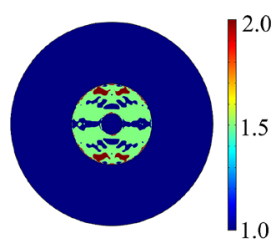

(a)

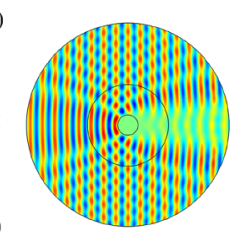

(b)

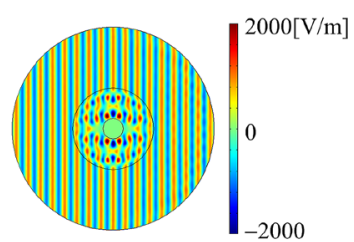

(c)
FIG. 3. (a) Optimized distribution of dielectric materials for Example 1 (red: dielectric material with $\varepsilon_{r}=2.0$; green: dielectric material with $\varepsilon_{r}=1.5$; blue: air). Total electric field without cloak (b) and with the optimized cloak (c).

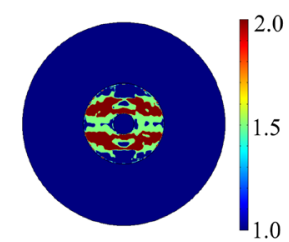

(a)

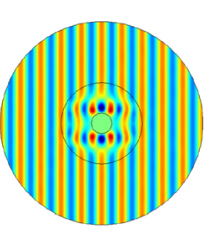

(b)

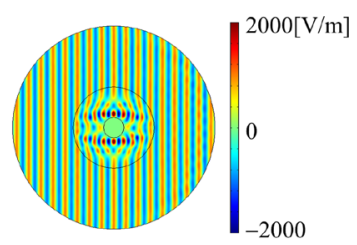

(c)
FIG. 4. (a) Optimized distribution of dielectric materials for Example 2 (red: dielectric material with $\varepsilon_{r}=2.0$; green: dielectric material with $\varepsilon_{r}=1.5$; blue: air). Total electric field of the optimized cloak at $1.5 \mathrm{GHz}$ (b) and at $2.5 \mathrm{GHz}(\mathrm{c})$.

that the material and air domains are perfectly separated and that the boundaries between materials are clear. Figure 3(b) shows the electric field without the cloak, and Fig. 3(c) shows the electric field of the optimized cloak during operation. As Fig. 3(b) shows, the electromagnetic waves are scattered by the cylindrical scattering object without the cloak, but scattering is much reduced when the cloak is operating, as shown in Fig. 3(c). The values of the objective functional without and with the cloak are $1.1 \times 10^{6}$ and $9.1 \times 10^{1}$, respectively. These results indicate that the optimization found an appropriate multimaterial dielectric configuration for a cylindrical electromagnetic cloak.

Next, we show the results of Example 2. In this numerical example, we find the optimized configuration in response to two incident wave frequencies, $1.5 \mathrm{GHz}$ and $2.5 \mathrm{GHz}$. The objective functional is formulated as follows, using the weighted sum method:

$$
F\left(\psi_{n}^{k}\right)=\sum_{p=1}^{2} \int_{\Omega_{\text {out }}} w_{p}\left(E_{z}^{p}-E_{z}^{p, \text { ref }}\right)^{*}\left(E_{z}^{p}-E_{z}^{p, \text { ref }}\right) \mathrm{d} \Omega,
$$

where $E_{z}^{1}$ and $E_{z}^{2}$ are the electric fields when the incident frequencies are $1.5 \mathrm{GHz}$ and $2.5 \mathrm{GHz}$, respectively. In this example, the weight $w_{p}$ is set to 1.0. Figure 4(a) shows the optimized configuration of dielectric materials. Figures 4(b) and $4(\mathrm{c})$ show the electric field of the optimized cloak during operation at $1.5 \mathrm{GHz}$ and $2.5 \mathrm{GHz}$, respectively. These results indicate that the optical cloak performs for electromagnetic waves at two different incident frequencies.

We now compare the performance of the optimization result using multiple materials with that when a single material is used. Figure 5 plots the objective functional value

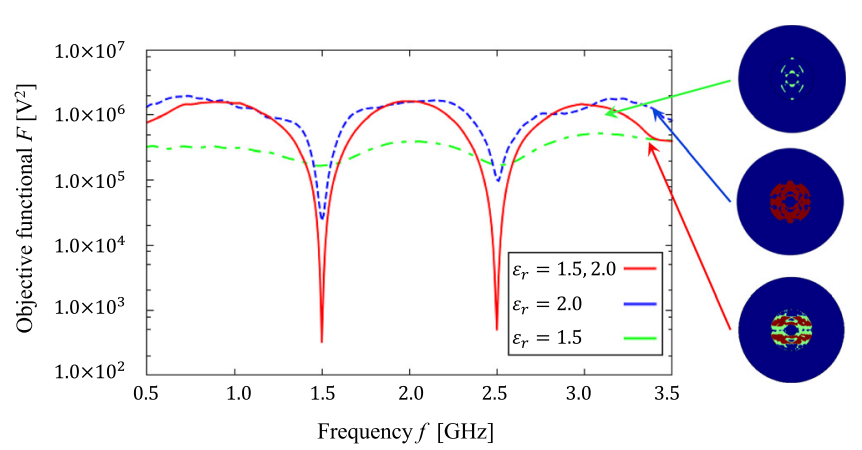

FIG. 5. Objective functional $F$ versus frequency $f$ at $\theta=0$ for the optimized cloak with multiple materials (red line) and a single material (blue and green lines). 


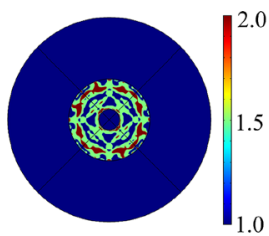

(a) (b)

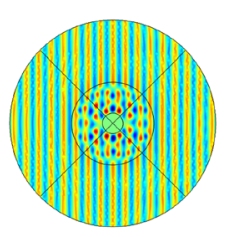

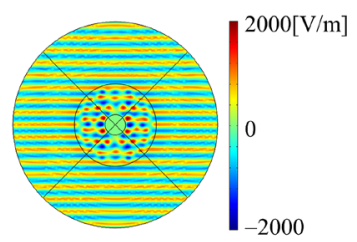

(c)
FIG. 6. (a) Optimized distribution of dielectric materials for Example 3 (red: dielectric material with $\varepsilon_{r}=2.0$; green: dielectric material with $\varepsilon_{r}=1.5$; blue: air). Total electric field of the optimized cloak for incident waves in the $x$ direction (b) and $y$ direction (c).

versus frequency for each configuration obtained when using multiple materials and a single material. This result reveals that the cloaking performance is the highest at the target frequencies when using multiple materials. Moreover, we can confirm that an optical cloak is realized for a wider frequency range when multiple materials are used. Therefore, the cloak design based on the use of multiple materials has better performance than a single material cloak design.

Finally, we present the result of Example 3. In this numerical example, we find the optimized configuration for incident waves arriving from different directions, up, down, left, and right. To reduce the calculation cost, we apply the symmetric design constraint proposed by Andkjær and Sigmund. ${ }^{15}$ Specifically, we introduce two symmetry planes separated by angles $\pi / 2$ and intersecting at the origin. The operating frequency is set as $2.5 \mathrm{GHz}$. Figure 6 (a) shows the optimized configuration of dielectric materials, and Figs. 6(b) and 6(c) show the total electric field of the optimized cloak for incident waves propagating in the $x$ and $y$ directions. These results indicate that the electromagnetic cloak operates for incident waves in four directions. Although the settings for this problem are the same as in Ref. 15, we can confirm that grayscales are completely absent. Figure 7 plots objective functional values versus the angle of incidence from $-\pi$ to $\pi$. The results reveal that the cloaking effect is the highest when using multiple materials at the targeted incident angles. Therefore, in this case as well, we confirm that a cloak employing multiple materials has better performance than that of a single material cloak design.

To conclude, we proposed a design method for an electromagnetic cloak comprised of multiple dielectric materials using a topology optimization method. We achieved the following:

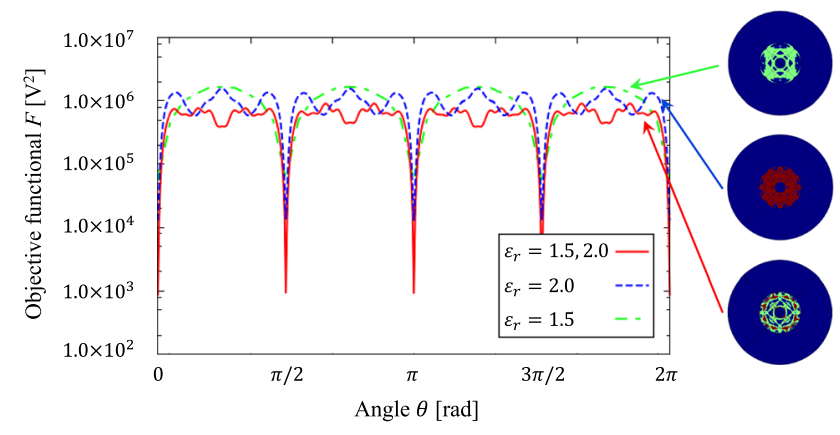

FIG. 7. Objective functional $F$ versus angle of incidence $\theta$ for the optimized cloak using multiple materials (red line) and a single material (blue and green lines).
(1) To obtain a clear optimized configuration for multiple materials, we applied a level set-based shape representation method for multiple materials to a topology optimization method.

(2) The design problem requirements were clarified, and an optimization problem was formulated based on the MMLS method.

(3) We explained how the topological derivatives were derived for multiple materials.

(4) Numerical examples of three optimization problems demonstrated that the optimization method found configurations of dielectric materials that function as electromagnetic cloaks.

(5) We confirmed that objective functional values became much smaller when designing with multiple materials than when a single material is used and demonstrated the superiority of a multiple-material approach.

${ }^{1}$ U. K. Chettiar, A. V. Kildishev, H.-K. Yuan, W. Cai, S. Xiao, V. P. Drachev, and V. M. Shalaev, "Dual-band negative index metamaterial: Double negative at $813 \mathrm{~nm}$ and single negative at $772 \mathrm{~nm}$," Opt. Lett. 32, 1671-1673 (2007).

${ }^{2}$ W. Sun, Q. He, J. Hao, and L. Zhou, "A transparent metamaterial to manipulate electromagnetic wave polarizations," Opt. Lett. 36, 927-929 (2011).

${ }^{3}$ M. Otomori, T. Yamada, K. Izui, S. Nishiwaki, and J. Andkjær, "A topology optimization method based on the level set method for the design of negative permeability dielectric metamaterials," Comput. Methods Appl. Mech. Eng. 237, 192-211 (2012).

${ }^{4}$ J. B. Pendry, D. Schurig, and D. R. Smith, "Controlling electromagnetic fields," Science 312, 1780-1782 (2006).

${ }^{5}$ U. Leonhardt, "Optical conformal mapping," Science 312, 1777-1780 (2006).

${ }^{6}$ M. Yan, Z. Ruan, and M. Qiu, "Scattering characteristics of simplified cylindrical invisibility cloaks,” Opt. Express 15, 17772-17782 (2007).

${ }^{7}$ Y. You, G. W. Kattawar, and P. Yang, "Invisibility cloaks for toroids," Opt. Express 17, 6591-6599 (2009).

${ }^{8}$ Y. Lee and D. Ahn, "Full-wave finite-difference time-domain analysis of the invisibility cloak mapped to a line segment with isotropic complementary media," J. Opt. Soc. Am. B 30, 2148-2156 (2013).

${ }^{9}$ C. Qian, R. Li, Y. Jiang, B. Zheng, H. Wang, Z. Xu, and H. Chen, "Transient response of a signal through a dispersive invisibility cloak," Opt. Lett. 41, 4911-4914 (2016).

${ }^{10}$ Y. Kim, I. Seo, I.-S. Koh, and Y. Lee, "Design method for broadband freespace electromagnetic cloak based on isotropic material for size reduction and enhanced invisibility," Opt. Express 24, 22708-22717 (2016).

${ }^{11}$ Y. Urzhumov, N. Landy, T. Driscoll, D. Basov, and D. R. Smith, "Thin low-loss dielectric coatings for free-space cloaking," Opt. Lett. 38, 1606-1608 (2013).

${ }^{12}$ D. Schurig, J. Mock, B. Justice, S. A. Cummer, J. B. Pendry, A. Starr, and D. Smith, "Metamaterial electromagnetic cloak at microwave frequencies," Science 314, 977-980 (2006).

${ }^{13}$ I. Smolyaninov, Y. Hung, and C. Davis, "Two-dimensional metamaterial structure exhibiting reduced visibility at $500 \mathrm{~nm}$," Opt. Lett. 33, 1342-1344 (2008).

${ }^{14}$ Y. G. Ma, C. Ong, T. Tyc, and U. Leonhardt, "An omnidirectional retroreflector based on the transmutation of dielectric singularities," Nat. Mater. 8, 639-642 (2009).

${ }^{15}$ J. Andkjær and O. Sigmund, "Topology optimized low-contrast alldielectric optical cloak,” Appl. Phys. Lett. 98, 021112 (2011).

${ }^{16}$ T. Yamada, H. Watanabe, G. Fujii, and T. Matsumoto, "Topology optimization for a dielectric optical cloak based on an exact level set approach," IEEE Trans. Magn. 49, 2073-2076 (2013).

${ }^{17}$ G. Fujii, H. Watanabe, T. Yamada, T. Ueta, and M. Mizuno, "Level set based topology optimization for optical cloaks," Appl. Phys. Lett. 102, 251106 (2013).

${ }^{18}$ M. Otomori, T. Yamada, J. Andkjaer, K. Izui, S. Nishiwaki, and N. Kogiso, "Level set-based topology optimization for the design of an electromagnetic cloak with ferrite material," IEEE Trans. Magn. 49, 2081-2084 (2013). 
${ }^{19}$ J. Andkjær, N. A. Mortensen, and O. Sigmund, "Towards all-dielectric, polarization-independent optical cloaks,” Appl. Phys. Lett. 100, 101106 (2012).

${ }^{20} \mathrm{~N}$. Heo and J. Yoo, "Dielectric structure design for microwave cloaking considering material properties," J. Appl. Phys. 119, 014102 (2016).

${ }^{21}$ M. P. Bendsøe and N. Kikuchi, "Generating optimal topologies in structural design using a homogenization method," Comput. Methods Appl. Mech. Eng. 71, 197-224 (1988).

${ }^{22}$ T. Yamada, K. Izui, S. Nishiwaki, and A. Takezawa, "A topology optimization method based on the level set method incorporating a fictitious interface energy," Comput. Methods Appl. Mech. Eng. 199, 2876-2891 (2010).
${ }^{23}$ L. Lan, F. Sun, Y. Liu, C. Ong, and Y. Ma, "Experimentally demonstrated a unidirectional electromagnetic cloak designed by topology optimization," Appl. Phys. Lett. 103, 121113 (2013).

${ }^{24}$ Y. Wang, Z. Luo, Z. Kang, and N. Zhang, "A multi-material level setbased topology and shape optimization method," Comput. Methods Appl. Mech. Eng. 283, 1570-1586 (2015).

${ }^{25}$ A. A. Novotny, R. A. Feijóo, E. Taroco, and C. Padra, "Topological sensitivity analysis," Comput. Methods Appl. Mech. Eng. 192, 803-829 (2003).

${ }^{26}$ A. Carpio and M. Rapun, "Solving inhomogeneous inverse problems by topological derivative methods," Inverse Probl. 24, 045014 (2008). 